\title{
Estimating philopatry and natal dispersal of microtine rodents through intensive live-trapping at nests of social groups
}

\author{
LOWELL L. GETZ \\ University of Illinois, Urbana, Illinois \\ BETTY MCGUIRE \\ University of Massachusetts, Amherst, Massachusetts \\ and \\ MARIA E. SNARSKI \\ University of Illinois, Urbana, Illinois
}

\begin{abstract}
Estimates of philopatry and natal dispersal of the prairie vole, Microtus ochrogaster, determined by intensive live-trapping at social group nests were assessed by comparison of estimates when a grid pattern within the area was also trapped, the periphery of the group home ranges was trapped, and when only a few and a large number of nests were being monitored. Several analyses confirmed that the measures of philopatry and natal dispersal were reliable. Although labor intensive, the protocol used is less so than any other system that provides comparable amounts of data, and it does not require expensive equipment.
\end{abstract}

A current active area of study among behavioral ecologists is determination of the mating system and social organization of small mammals. Philopatric behavior and natal dispersal are important components of the type of mating system and resulting social organization displayed by a species. An understanding of environmental and social conditions associated with philopatry is especially important in determining the advantages of forming communal groups that include philopatric offspring. Accordingly, a number of hypotheses have been proposed to explain selective factors associated with philopatric behavior (Brown, 1974; Emlen, 1982; Koening \& Pitelka, 1981; Lott, 1984; Stacey \& Ligon, 1987). In order to test these hypotheses, it is necessary to distinguish between philopatric animals and those that disperse from the natal nest.

Unlike birds, which are often conspicuous to the observer, most small mammals are secretive and/or nocturnal in habits, making observation of dispersal and location of the natal nest difficult. The only direct means to monitor location of individuals involves radiotelemetry. However, this is not a practical technique for the study of most small mammal species; available radio transmitters are too large to place in or on juveniles, and the short life of the batteries requires that transmitters be replaced at frequent intervals. Furthermore, too few individuals can be monitored concurrently to provide ample sample

This research was supported by National Institutes of Health Grant HD 09328 and National Science Foundation Grant DEB 78-25864. Correspondence should be addressed to L. L. Getz, Department of Ecology, Ethology, and Evolution, University of Illinois, 505 S. Goodwin Ave., Urbana, Il 61801 (e-mail: lowell_getz@qms1.life.uiuc.edu). sizes. All residents of each social group must be monitored. Owing to a high mortality rate of juveniles, $70 \%$ $80 \%$ die before becoming old enough to be categorized either as philopatric or as a natal disperser. Thus, an inordinately large number of young would have to be fitted with transmitters to obtain adequate data. The social group nests would have to be live-trapped at frequent intervals to ensure all young were fitted with transmitters as soon as they emerged from the natal nest and to replace dead radios on all residents. This would be in addition to the major effort required to maintain an adequate radiotelemetry monitoring schedule.

Accordingly, most studies of natal dispersal of small mammals involve indirect means of estimating philopatry and natal dispersal, primarily intensive live-trapping (Boonstra, Krebs, Gaines, Johnson, \& Craine, 1987; Hilborn, 1975; Lambin \& Krebs, 1991; Ostfeld, 1986). Most such studies involve trapping the study area in a grid pattern. Animals first captured below a designated body mass are assumed to be juveniles that have just emerged from the natal nest. In some analyses, the natal nest is assumed to be located at the station where the animal was first captured. Subsequent captures at other grid stations are interpreted to represent dispersal from the natal nest. These assumptions are subject to bias. The arbitrary body mass designation often is so large that, while the animal most likely has been born into the study population (rather than being an immigrant from another population), it may have already dispersed from the natal nest (Dueser, Rose, \& Porter, 1984; Tamarin, 1984). Even if the animal has not dispersed, the natal nest may be some distance from the station at which the animal was first captured. Since na- 
tal dispersal may involve short distances, estimates of the proportion dispersing, as well as the distances dispersed, may be inaccurate. Use of a sufficiently small grid interval to reduce these biases results in an excessive amount of trapping effort and still does not provide the precise location of the natal nest. Also, it does not confirm actual residency of an individual at a given nest.

In order to determine patterns of natal dispersal and philopatry in small mammals, it is necessary that we first determine the exact location of the natal nest and then continuously monitor all residents. In this paper, we describe a means of meeting these requirements and provide an assessment of the accuracy of our categorization of individuals either as philopatric or as having dispersed from the natal nest. Populations of the prairie vole, Microtus ochrogaster, were studied.

\section{METHOD}

\section{Study Areas}

The study was conducted in two adjacent one-hectare alfalfa (Medicago sativa) fields within the University of Illinois Biological Research Area (Phillips Tract), $6 \mathrm{~km}$ northeast of Urbana, IL. The first area was used as a study site from October 1980 through July 1984; the second area was used from June 1983 through May 1987. (See Getz, McGuire, Hofmann, Pizzuto, \& Frase, 1990, for a description of the study areas.)

\section{Field Procedures}

To determine the social system within the study populations, we monitored the nests of all adult females. Females to be located were identified from three trapping regimes: (1) twice-monthly live-trapping with traps positioned in a 10-m grid pattern, (2) twice-weekly monitoring of social group nests (see below), and (3) trapping three times a month at the periphery of social group home ranges (McGuire, Pizzuto, \& Getz, 1990). In all, three regimes multiple-capture live traps were used.

The entire grid was trapped for 3 days each month; traps were checked at 2130,0800 , and $1500 \mathrm{~h}$ daily, from Tuesday evening through Friday afternoon. Two weeks following the 3-day trapping session, those grid traps that were more than $15 \mathrm{~m}$ from known nests were set Wednesday afternoon and checked on the above schedule through Friday morning.

Nests of females were located by use of ultraviolet reflective powder (Lemen \& Freeman, 1985). After a nest was located, four or five traps were placed near the burrow openings to underground nests or in runways leading to surface nests. Traps at each nest were set for two 28-h periods each week (so long as the nest was occupied) and for 2 weeks following disappearance of the last resident. Cracked corn was used as bait. We set the traps shortly after sunrise on Monday and checked them at 2- to 4-h intervals until midnight and twice on Tuesday morning, approximately seven times over the 28 -h period. The trapping schedule was repeated Thursday morning through Friday morning. This regime of monitoring nests was maintained throughout the study except during the week of monthly grid-trapping when traps at nests were set only Monday through Tuesday.

From March 1986 through May 1987, six to eight traps were placed in a circle approximately $5 \mathrm{~m}$ from the nest (peripheral trapping) of an average of $10(6-19)$ social groups per week. The traps were set at $1100 \mathrm{~h}$ Tuesday and checked at 0900, 1500, and $2100 \mathrm{~h}$ through Wednesday night.

When first captured, voles were individually marked by toe-clipping and weighed. At each capture, location, animal number, sex, and reproductive condition were recorded. Weights of juveniles were used to estimate age at first capture (by reference to weights of animals of known age in a laboratory colony). Data are presented for the periods of late October through December 1980, mid-May through December 1981, and late February 1982 through May 1987 . Only nests that were occupied for at least 14 days were included in data analyses.

\section{Definitions}

Philopatry, as used in this paper, refers to individuals that remain at the natal nest until death. Only those individuals that survived beyond 30 days, the age at which most are capable of becoming reproductive, were used in our analysis of philopatry. Natal dispersal is defined as (1) capture of an animal at least one home range diameter $(20 \mathrm{~m})$ away from the natal nest and not at the nest again, (2) forming a breeding unit, (3) joining another established social group, or (4) leaving the nest and wandering within the study area until disappearing from the population through either mortality or emigration. We assume that when an individual disappeared from the natal nest and was not captured again, either within the study area or in an adjacent area, it had died while a resident of the natal nest. Employing the above protocol, we estimated that $72.2 \%$ of the 587 young for which we knew the location of the natal nest were philopatric. Thus, only $27.8 \%$ were recorded as having dispersed from the natal nest.

Even though we live-trapped all nests or the grid within the study areas twice weekly, failure to capture an animal that had disappeared from the nest is only indirect evidence of mortality while still living at the nest. An individual could have dispersed from the nest and died in unfamiliar surroundings before encountering a trap at another nest or grid station. Furthermore, repulsion of unfamiliar animals from a nest by residents of a social group (Getz \& Hofmann, 1986) may tend to prevent capture of dispersers at other nests, thus increasing the probability of mortality before being captured. The analyses involved comparison of known dispersal (and, in turn, presumed nest mortality) (1) immediately before and after a grid-trapping session was conducted, (2) when trapping was also conducted at the periphery of the home range of a large number of social groups, and (3) when very few nests were being trapped relative to when a large number of nests (including substantial numbers of nests with no actual residents) were being trapped.

\section{RESULTS}

\section{Before and After Grid Trapping}

As indicated above, the trapping regime for the 1 week each month the study areas were trapped at a $10-\mathrm{m}$ grid interval was as follows. Monday morning through Tuesday morning, all nests were monitored seven times, with the last check at $0930 \mathrm{~h}$ Tuesday. The grid traps were set at $1500 \mathrm{~h}$ Tuesday and checked through Friday afternoon (a total of nine checks). If animals that disappeared from the nest had actually dispersed, one would anticipate catching more animals after they had disappeared from the nest on a Monday-Tuesday prior to a Tuesday-Friday gridtrapping of the area than when a Monday-Tuesday nesttrapping session was followed by a Thursday-Friday nesttrapping. The grid traps were evenly distributed throughout the area to intercept dispersing animals, and the potential problem resulting from repulsion of unfamiliar animals from traps placed directly at a nest would be avoided. If apparent nest mortality resulted from an inability to intercept dispersing animals before they succumbed to mortality outside the natal home range, we would expect to record more dispersal during the week of grid-trapping than during other weeks.

To test this assumption, natal dispersal of young that were last captured at their natal nest Monday-Tuesday prior to the Tuesday-Friday grid-trapping was compared with that of animals last caught the following MondayTuesday trapping, which was followed by ThursdayFriday trapping at the nests. Only data from the MondayTuesday following the grid-trapping week were used in 
the analyses to reduce temporal effects on dispersal. The capture data for the months of April-October (the months most young were born) of 1982-1986 and April and May 1987 were analyzed. There was no significant difference between dispersal values for these 2 weeks of each month: $29.9 \%(n=137)$ and $27.6 \%(n=116)$, respectively; $\chi^{2}(1)=0.073, p>.787$. There was no difference in dispersal of males $(27.9 \%, n=61$, and $26.1 \%, n=46)$ or females $(31.6 \%, n=76$, and $28.6 \%, n=70)$ for the 2 weeks.

Once a month, the grid traps that were more than $15 \mathrm{~m}$ from a known nest were set at $1500 \mathrm{~h}$ on Wednesday and checked through Friday morning (partial grid-trapping). As per above, compilations of known dispersers that disappeared from their natal nest Monday-Tuesday prior to the partial grid-trapping were compared with those that disappeared the next Monday-Tuesday, which was followed by Thursday-Friday nest-trapping. Of the $\mathbf{4 6}$ young disappearing before a partial grid-trapping, $32.6 \%$ were known dispersers (trapped at grid stations or at another nest); $30.0 \%$ of the $\mathbf{4 0}$ disappearing the next Monday-Tuesday were known dispersers $\left[\chi^{2}(1)=0.001\right.$, $p>$.97].

\section{Peripheral Trapping}

If residents repulsed unfamiliar dispersing animals from the traps at the nest, we would expect higher known dispersal when peripheral traps were set than when such traps were not set. We first compared only subsequent captures of animals that disappeared from social groups included in the peripheral study the Monday-Tuesday prior to the start of trapping at the periphery. Only 13 individuals disappeared from the specific social groups included in the peripheral study; 2 (15.4\%) were recaptured elsewhere in peripheral traps. We then calculated known dispersal of young that disappeared from the natal nest of all social groups, whether part of the peripheral study or not, during the period of the peripheral trapping. Of a total of 95 young that disappeared from the natal nests, only 5 (5.3\%) were subsequently captured in peripheral traps. Both such estimates are well below the $27.8 \%$ recorded as natal dispersers for the main data set.

\section{Small Versus Large Number of Nests Being Trapped}

We would expect to have captured more dispersing animals when a large number of closely spaced nests were trapped than when fewer, more widely dispersed nests were trapped. Owing to the greater distance between nests when few were present (the nests were not clumped within the study areas), there would be higher probability for mortality of dispersers prior to encountering traps at other nests than when a large number of nests were being trapped.

During the summer, most social groups formed one or more surface nests within their home ranges. Although these normally did not constitute the primary nest of the social group (primary nests were usually underground), a few were the primary nest of the group. At high densities, such surface nests were close together so that it was difficult a priori to establish which was the primary nest of a social group. Accordingly, all nests were trapped until the group disappeared; the primary nest was identified when the capture data were analyzed. At low densities, it was easier to determine the primary nest of a social group; few secondary surface nests were trapped at these times. Furthermore, our monitoring protocol called for us to continue to trap a given nest for 2 weeks after the residents seemed to have disappeared from the nest to ensure that they had actually done so. Thus, at high densities, not only was the distance between nests small but a large number (frequently at least $50 \%$ ) of those nests being trapped at any given time were not inhabited; there would be no exclusion of dispersers from the latter traps. At low densities, very few uninhabited nests were trapped.

Comparisons of known dispersal when a large number of nests (at least 100) were trapped and when only a few nests (no more than 25) were trapped should indicate greater dispersal during the former periods, if our protocol underestimated natal dispersal. Such was not the case. Although not statistically significant, known dispersal was actually slightly higher $(33.3 \%, n=39)$ when few nests were trapped than when large numbers of nests were trapped $(20.6 \%, n=92)$. Known dispersal also showed the same trend when calculated for those periods when at least 100 nests, $26-99$ nests, and no more than 25 nests were trapped-18.0\% $(n=61), 30.9 \%(n=42)$, and $37.5 \%(n=24)$ known dispersal, respectively. This analysis assumes no major effect of population density on natal dispersal; none was observed.

\section{DISCUSSION}

Although the above estimates of natal dispersal and, in turn, nest mortality are also indirect, they eliminate many of the potential biases presented by nest-trapping alone. All suggest that when an animal disappears from the natal nest and is not caught elsewhere in the study area, it has died while a resident of the nest. If a significant proportion of these animals had actually dispersed, a comparison of dispersal estimates from the above trapping protocols should have provided at least some evidence of such. None of the data suggested undetected dispersal. We therefore conclude that our definition of mortality while a resident of the natal nest is reasonably accurate.

There is still the possibility that animals could have dispersed from the natal nest and emigrated immediately, without being caught within the study area. However, most of the suitable Microtus habitat adjacent to the two study areas was live-trapped monthly as part of a continuing demographic study of microtine populations (Getz et al., 1987). Only $5.0 \%$ of the total young known to have survived to trappable age were recovered in other study sites; $1.7 \%$ of these had been recorded as dispersers within the study area before they moved into the other areas. Thus, dispersal out of the two study areas was very low and probably did not affect our results.

The above-proposed protocol for estimating philopatry and natal dispersal is more reliable than and as time effective as previous methods employing only grid-trapping or radiotelemetry. Our system allows for (1) precise location of the natal nest, (2) identification of all residents of the natal nest, (3) determination of the proportion of offspring that are philopatric and those that are natal dispersers, and (4) precise determination of the distance dispersed for those individuals that leave home 
and form or join a new group. Although our system of live-trapping is labor intensive, it is less so than using radiotelemetry to obtain a comparable amount of data, and it does not require expensive equipment.

\section{REFERENCES}

Boonstra, R., Krebs, C. J., Gaines, M. S., Johnson, M. L., \& Craine, I. T. M. (1987). Natal philopatry and breeding systems in voles (Microtus spp.). Journal of Animal Ecology, 56, 655-673.

Brown, J. L. (1974). Alternate routes to sociality in jays-With a theory for the evolution of altruism and communal breeding. American Naturalist, 14, 63-80.

Dueser, R. D., Rose, R. K., \& PorTer, J. H. (1984). A body-weight criterion to identify dispersing small mammals. Journal of Mammalogy, 65, 727-729.

EmLEN, S. T. (1982). The evolution of helping: I. An ecological constraints model. American Naturalist, 119, 29-39.

Getz, L. L., \&ofmanN, J. E. (1986). Social organization in freeliving prairie voles, Microtus ochrogaster. Behavioral Ecology \& Sociobiology, 18, 275-282.

Getz, L. L., hofmann, J. E., Klatt, B. J., Verner, L., Cole, F. R., \& LiNDROTH, R. L. (1987). Fourteen years of population fluctuations of Microtus ochrogaster and $M$. pennsylvanicus in east central Illinois. Canadian Journal of Zoology, 65, 1317-1325.

Getz, L. L., McGuire, B., Hofmann, J., Pizzuto, T., \& Frase, B. (1990). Social organization and mating system of the prairie vole, Microtus ochrogaster. In R. H. Tamarin, R. S. Ostfeld, S. R. Pugh, \& G. Bujalska (Eds.), Social systems and population cycles in voles (pp. 69-80). Basel: Birkhauser.
Hilborn, R. (1975). Similarities in dispersal tendency among siblings in four species of voles (Microtus). Ecology, 56, 1221-1225.

Koening, W. E., \& Pitelka, F. A. (1981). Ecological factors and kin selection in the evolution of cooperative breeding in birds. In R. D. Alexander \& D. W. Tinkle (Eds.), Natural selection and social behavior: Recent research and new theory (pp. 261-280). New York: Chiron.

LAMBIN, X., \& KREBS, C. J. (1981). Spatial organization and mating system of Microtus townsendii. Behavioral Ecology \& Sociobiology, 28, 353-363.

Lemen, C. A., \& Freeman, R. W. (1985). Tracking mammals with fluorescent pigments: A new technique. Journal of Mammalogy, 66, 134-136.

LoTT, D. F. (1984). Intraspecific variation in the social systems of wild vertebrates. Behaviour, 88, 266-325.

McGuire, B., Pizzuto, T., \& Getz, L. L. (1990). Potential for social interaction in a natural population of prairie voles (Microtus ochrogaster). Canadian Journal of Zoology, 68, 391-398.

OSTFELD, R. S. (1986). Territoriality and mating system of California voles. Journal of Animal Ecology, 55, 691-706.

STACEY, P. B., \& Ligon, J. D. (1987). Territory quality and dispersal option in the acorn woodpecker, and a challenge to the habitat-saturation model of cooperative breeding. American Naturalist, 130, 654-676.

TAMARIN, R. H. (1984). Body mass as a criterion of dispersal in voles: A critique. Journal of Mammalogy, 65, 691-692.

(Manuscript received January 20, 1992.) 